\title{
Decision Support System in Healthcare Industry
}

\author{
K. Rajalakshmi \\ Asst. Professor \\ MBA Dept. \\ J.J. College of Engineering and \\ Technology, \\ Trichy-09.Tamil Nadu, India
}

\author{
Dr. S. Chandra Mohan \\ Professor \\ Alagappa Institute of \\ Management Studies, \\ Karaikudi, \\ Tamil Nadu, India.
}

\author{
Dr.S.Dhinesh Babu \\ Asst. Professor \\ MBA Dept. \\ Government Arts College, \\ Paramakudi, \\ Tamil Nadu, India.
}

\begin{abstract}
In this paper we propose Decision support system implementation for health care system with the use of decision support system. The system integrates the input data from relevant sources into an autonomous data warehouse, while the client front- end ensures adequate output presentation to the user so as reveal significant details and dependencies. Applying the system as an "on -the -fly" decision-support facility for the policy-makers has resulted in significant acceleration of planning procedure, raised the overall awareness with respect to the underlying methodology and, ultimately, enabled more efficient academic administration.
\end{abstract}

\section{Keywords}

Healthcare, Data warehouse, Doctors, Data mining.

\section{INTRODUCTION}

The healthcare industry generates massive amount of data. Information Technology (IT) is, therefore, used extensively to capture and transfer information. The article explains the role played by [1] decision support system which is a 'computerbased system that aids in the process of decision-making' in order to ensure correct diagnosis of any illness.

Healthcare industry is growing fast by using IT to automate its many processes like transaction, inventory keeping and maintaining records, thus eliminating mundane and repetitive processes. [2] Healthcare is the management, prevention and the treatment of illness and the main aim is to provide clean and effective services that lead to the preservation of mental and physical well-being of humans and animals.

The medical diagnosis of an illness can be done in many ways; from the patient's description, physical examination and/or laboratory tests. After the diagnosis is done by the doctor, treatment is given keeping in mind drug reactions and allergies. However there is always a chance of wrong diagnosis which may lead to drug reactions and allergies in a patient and may lead to life threatening situations. To address these points, one of the IT systems that help the healthcare professionals in problem solving during medical diagnosis is the Decision Support System (DSS).

DSS is also defined as "an interactive, flexible and adaptable computer-based information system" and is developed to provide solution for non- structured management problem. It utilizes data, provides an easy-to-use interface and allows for the decision-makers' own insights. In healthcare domain, DSSs are also commonly known as Clinical Decision Support System (CDSS).[3] It is defined as a software that helps in clinical decision-making, in which the "characteristics of an individual patient are matched to a computerized clinical knowledge base and patient-specific assessment or recommendations are then presented to the clinical or the patient for a decision". The main advantage of this system is that it helps to improve patient care and efficiency of the healthcare providers. (4). "Act of providing clinicians, patients and other health care stakeholders with pertinent knowledge and/or person-specific information, intelligently filtered or presented at appropriate times, to enhance health and health care" (5) CDS include providing reminders of required chronic disease management services to clinicians within an electronic health record system, providing screening recommendations to patients within a web-based personal health record system, and delivering patient-specific recommendations to clinicians within a computerized provider order entry system.

\subsection{Data warehousing}

A data warehousing is a [6] "a subject oriented, integrated, non volatile and time-variant collection of data in support of management's decisions". The main objective is to bring together the various data which are distributed in transactional heterogeneous database into a single collection. The centralization of voluminous amount of data allows the discovering of trends and provides hidden information that eases managers' decisions.

\subsection{Data Mining}

Data warehousing and Knowledge Management can contribute a lot to decision support system in Health care procedures. [7] Data mining is also a core step, which results in the discovery of hidden but useful information from massive database and health care operation which involves pragmatic use of database and the techniques of data mining and data warehousing for taking various decisions for the benefits of the society.

\section{DSS IN HEALTHCARE: AN OVERVIEW}

Decision making in healthcare is primarily done in two areas. The first area (lower level) involves patient management, diagnosis and treatment, record keeping, finance and inventory management. The other area involves higher level decision 
making that gives the hospital a competitive edge. The shareholders that play an important role in lower level decision making constitute of doctors and nurses. One such DSS that encompasses all functions from patient management to inventory management was designed by UK General Practice and is named PRODIGY (Prescribing Rationally with Decision support In General Practice Study). It provides access to clinical knowledgebase on the best evidence available about conditions and symptoms managed by primary healthcare professionals. Aimed at nurses, pharmacists, and patients, it provides full text guidance, quick reference guides, patient's information leaflets, information on drugs, and self help contacts.

DSS helps the finance department of the hospital keep track billing, accounts receivable, payroll and accounts payable. This system also helps to maintain insurance policy of the patient, insurance claims and different payment options. One of the decision support systems that help in this aspect is provided by med sphere. It is a corporation that provides different modules for decision support system in healthcare. It leverages its tightly integrated functionally and captures required financial information starting from registration through the patient encounter till his discharge. Supply chain management, collection processing, and numerous other related functions are immediately to check the financial pulse of the organization by this system.

DSS helps in diagnosis by providing a dictionary of health problems to the clinicians or by displaying background information about specific patients. It also provides assistance with diagnosing a patient's condition, guide to proper drug usage, reminders to administer preventive services to give patients at specific time. One of the products in this domain is Isabel, a web-based system that has been interfaced with electronic patient/medical record. It has two component systems: Isabel Diagnosis Reminder System (IDRS) and Isabel Knowledge Mobilizing System (IKMS). IDRS gives the doctors and physicians a likely list of diagnoses for given set of clinical features that are based on symptoms, signs and result of tests. IKMS has a dictionary of 10,000 diagnostic categories which help the doctors and physicians to do concepts search instead of keyword search in the in-built knowledge silos present in the system.

\section{DESIGN OF DSS}

The design of DSS is based on the stake holders involved and the type of decisions it has to be made. When designing health care DSS, organization design like decision making procedures, strategic planning process and governance structure should be kept in mind. A case study Penn CARE and Hendry Ford health system revealed that organization design consistency like cultures, strategic planning process, governance structure and decision making procedures is necessary for any system to be effective. It is also seen in many literature that effectiveness of the DSS is dependent on the model or methodology used to design the system. Currently, there are three approaches: Clinical algorithms, mathematical and computational approaches and heuristics approaches.

Another stream of research in DSS strongly believed that medical knowledge consisting of various patterns of any system that identifies these patterns was more effective. Neural network methodologies were used to design DSS and the use was limited to laboratory applications or medical imaging applications. Literature review has revealed that neural network systems are quite capable of providing the significant planning of patient care, especially in determining the length of stay of a patient and mortality rate. However, extensive research and resources are required to design and implement DSS by this methodology.

Another popular heuristic approach is data mining techniques. These techniques are being used to identify pattern or rules about various quality problems. DSS is built based on the data that are derived by data mining techniques and is effective in reducing cost incurred by the hospital by preventing adverse medical events and improving quality of care. Another system that improves quality and delivery of health care services is Strategic Healthcare Decision Support Services (SHDS) which is a synergy between knowledge management and data mining techniques.

\section{ISSUES}

DSS in healthcare is seen to improve quality, safety and efficiency of the hospital and patient care. Other advantage is seen in reduction of medication adverse drug events. However there are many challenges

Cost of the CDSS investment is also complex and there is ensuing debate about what should be included or excluded while calculating the cost and the benefit of this system.

Apart from cost, it is felt the quality and speed of decision making can be improved if emphasis is made on addressing the five major blocks. These blocks occur when stake holders are analyzing, have many advices and alternatives, cannot reach a unanimous decision and have no coordination in their activities. One of the ways to avoid spending time on analyzing is to process information quickly which could be done by facilitating the use of e-mail and face to face meeting or through group DSS. Stake holders have the tendency to get stuck over one alternative and have a conflict over the other alternative which can be avoided by having a multi disciplinary group brain storming session also, voting and appointment of counselors and facilitators would improve the quality of decision making.

The above mentioned blocks is also one of the problems that affect the adaptation of DSS although quite beneficial. DSS is not wide spread in its use the major reason being the complexity of these systems and vast knowledge base that need to be mined to reach a decision. As the doctors and nurses have to deal with various complex diagnoses, it becomes time consuming for them to adopt new systems. That is why, DSS, especially the clinically support system although perceived effective and efficient are not accepted widely due to time constraint and complexity. Other factors that account low adaptation of DSS or health administrators' focus on financial systems, government policies and physicians resistance to these systems. 


\section{DSS}

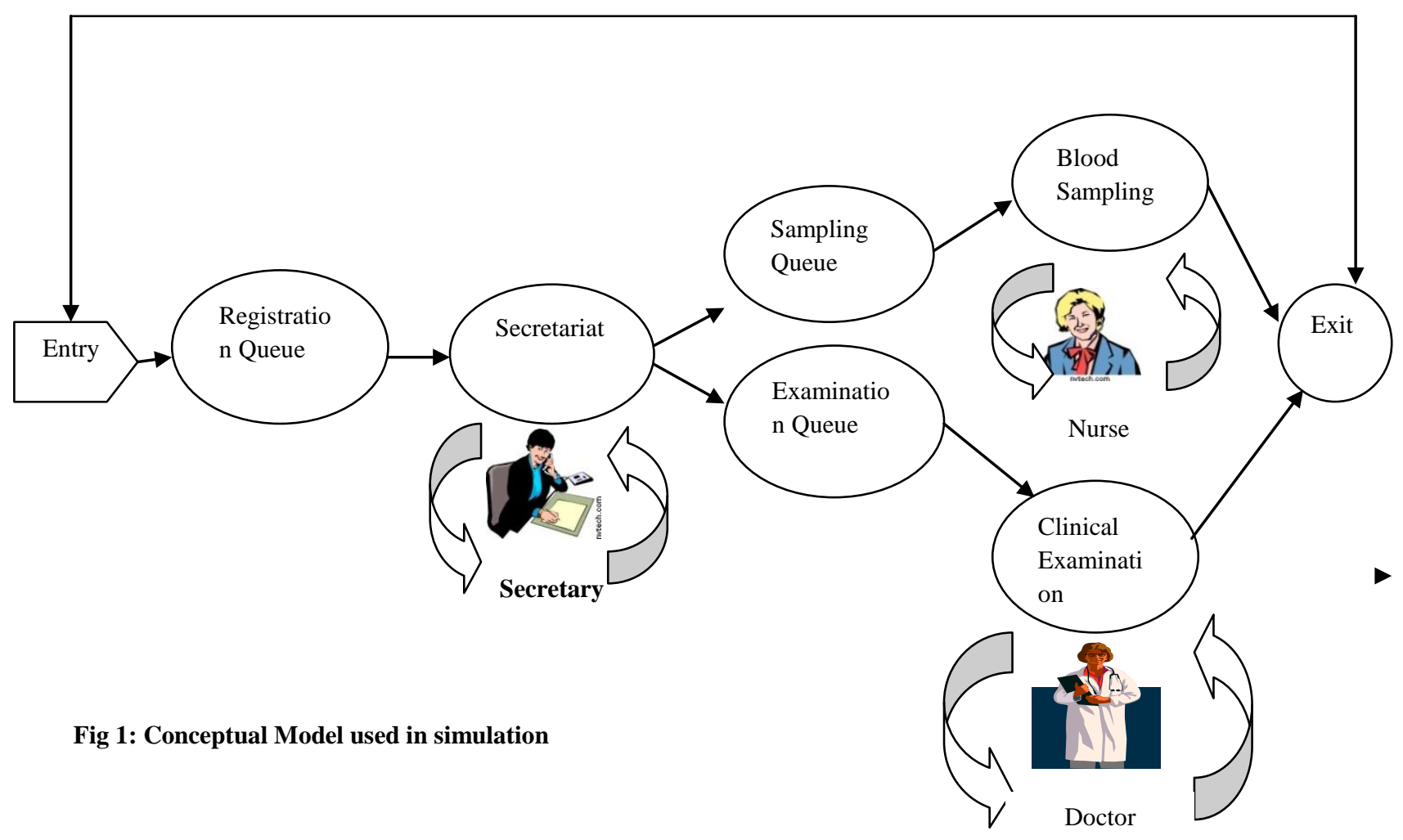

\section{CONCLUSION}

DSS has emerged as one of the dominant IT technologies for decision making in healthcare. However, these has resulted in many technologies being used in the design of DSS and has thus created various platforms in healthcare domain. Those are difficult to integrate. Literature review has revealed that it is vital that all the systems are integrated and towards these initiatives various integration projects like synergy extranet (SynEx) and health level seven have been started. Although factors like complexity and adoption rate persist, changes in clinical economics more computer literacy in general population and most importantly, changes in government policies and increased support of clinical computing suggest that the wave may break in the next decade.

\section{REFERENCES}

[1] Tan, J\& sheps ,B.(1998) Health decision support systems, Jones \&Bartlett publishers

[2] Wickramasinghe, N.\&Geisler, E.(2008).Encyclopedia of health care information systems, information science

[3] Perrealult, L.E.\&Metzger ,Jane B.(1999). “A pragmatic frame work for understanding clinical decision support system".
[4] Osheroff JA, Teich JM, Middleton B, Steen EB, Wright A,Detmer DE: A roadmap for national action on clinical decision support. J Am Med Inform Assoc 14: 141-145, 2007

[5] Osheroff JA, Pifer EA, Teich JM, Sittig DF and Jenders RA:Improving Outcomes with Clinical Decision Support: An Implementer's Guide, Chicago, IL, Health Information and Management Systems Society, 2005

[6] W.H.INMON, “ Building the Data Warehousing”, 2nd edition, John Wiley\& sons, New York (1996)

[7] Gramatikov martin "Data Mining Techniques and the decision making Process in the Bulgarian Public administration". 